\title{
Stable Multi-Particle Systems and Application in Multi-Vehicle Path Planning and Coverage
}

\author{
Ali Ahmadzadeh, Ali Jadbabaie, Vijay Kumar and George J. Pappas
}

\begin{abstract}
We present a path planning algorithm for cooperative coverage using a set of nonholonomic autonomous vehicles. The paper describes our overall framework and algorithm for the computation of trajectories that maximize spatio-temporal coverage while satisfying hard constraints such as collision avoidance and specifications on initial and final positions. Our approach is based on approximation of the trajectories of vehicles using sequence of waypoints and treating each way point as a moving particle in the space. We defines interaction forces between the particles such that the resulting multi-particle system will be stable, moreover, the trajectories generated by the waypoints in the equilibria of the multiparticle system will satisfy all of the hard constraint while generating a suboptimal solution to the coverage problem.
\end{abstract}

\section{INTRODUCTION}

In this paper we consider the problem of multi-vehicle path planning and coverage with spatio-temporal boundary conditions. Each vehicle is modeled as a nonholonomic vehicle with a bounded curvature trajectory which is known as Dubin's vehicle in the literature [1]. In this paper we present an approximate solution to this problem which can be generated in polynomial time. With the assumption of constant speed the trajectory of each vehicle can be considered as a fixed length bounded curvature curve that connects the initial and final positions of the vehicle. In this paper we approximate this bounded curvature curve by a polygonal curve and each node of this polygonal curve represents a waypoint of the vehicle. We find necessary and sufficient conditions for bounded curvature polygonal curves. The polygonal curve is constructed by treating each node of the curve as a moving particle in the space. We define interaction forces between the particles such that the resulting multi-particle system would be stable; moreover, the polygonal curve generated by the waypoints in the equilibria of the multi-particle system will satisfy all of the necessity conditions for bounded curvature curve as well as other hard constraints such as collision avoidance.

Most of prior work in the area of coverage problem concentrated on sensor network problem [2]. The cooperation of multiple sensors is often achieved by choosing different sensors (or sensor modes) for different tasks (targets) at different times and motion planning is not involved in these

\footnotetext{
$\dagger$ This research is supported in parts by the following grants: Army Research Office DURIP Grant W911NF-04-1-0148, ARO-MURI W911NF05-1-0381, ONR/YIP N00014-04-1-0467, ONR N00014-06-1-0436, and NSF-ECS-0347285.

Authors are with GRASP Laboratory, University of Pennsylvania, Philadelphia, PA 19104,USA

\{aliahmad, jadbabai, kumar, pappasg\} @grasp.cis.upenn.edu
}

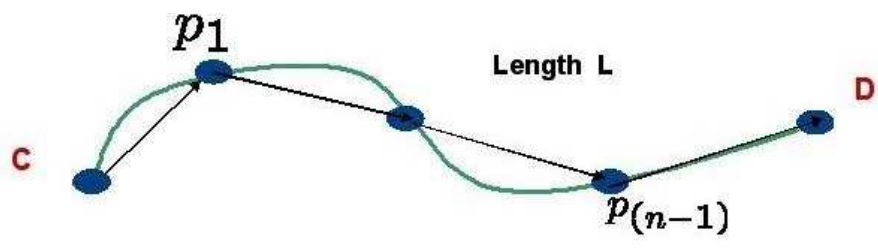

Fig. 1. Approximation of a smooth curve by a polygonal curve.

problems. Cortes et al. [5] studied the multi-sensor localization problem in a polygonal environment and developed a gradient-descent algorithm for optimal coverage and sensing policies. Each sensor agent is expected to converge to its optimal location and stay there. Enrigh et al. [6] considered the problem of visiting stochastically-generated targets in a planar bounded region with the objective of minimization of the expected waiting time between the appearance of a target, and the time it is visited. In [10] and [11] the coupling between flight path and the camera field of view is studied and an Integer Programming(IP)-based algorithm for time-critical cooperative surveillance using a set of UAVs is introduced.

The paper is organized as follows, first we find the conditions for bounded curvature polygonal curves. Then we try to solve the path planning problem in a most basic form which is to find a bounded curvature curve connecting two points. Then we add multi-vehicle scenario which involves collision avoidance. Eventually we consider the multi-vehicle coverage problem.

\section{Polygonal Curve Approximation}

Since each vehicle travels with constant speed and the time horizon of each vehicle is fixed; therefore, the length of the path that the vehicle travels is fixed. In the rest of the paper, we assume that the trajectory of each vehicle is a fixed length bounded curvature curve with a given initial and final configuration.

Consider a bounded curvature curve $\gamma$ with length $L$, connecting points $C$ and $D$. We can approximate the curve using finite number of vertices each lying on the curve, connected by straight edges. The resulting polygonal curve $\gamma_{p}$ is represented by its ordered vertices $p_{0}, p_{1}, \ldots, p_{n} \in \mathbb{R}^{2}$, where $p_{0}=C, p_{n}=D$ and $p_{i} p_{i+1}$ is the line segment connecting $p_{i}$ to $p_{i+1}$ (Fig. 1).

The length of polygonal curve $\gamma_{p}$ is given by:

$$
\operatorname{len}\left(\gamma_{p}\right)=\sum_{i}^{n}\left\|p_{i}-p_{i-1}\right\| \simeq L
$$




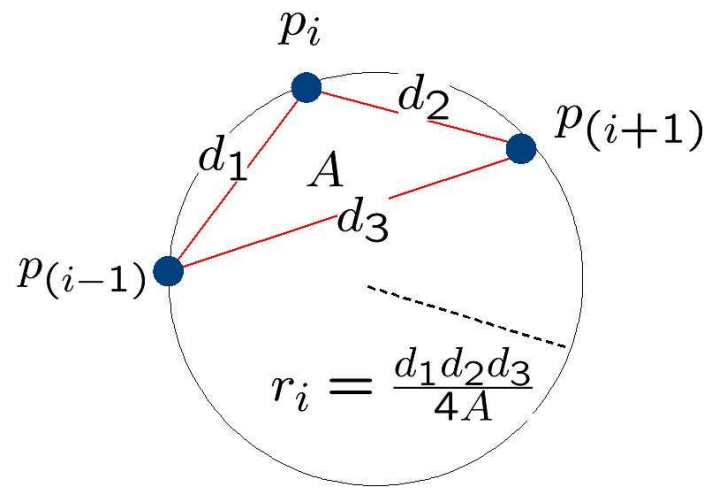

Fig. 2. Approximation of a smooth curve by a polygonal curve.

With the assumption of equidistance edges with length $d$, we get:

$$
d=\left\|p_{i}-p_{i-1}\right\| \simeq L / n
$$

In order to approximate the curvature of the polygonal curve at point $p_{i}$, we can use the circle passing through the points $p_{i-1}, p_{i}$ and $p_{i+1}$, as an approximation to the osculating circle to the curve at $p_{i}$ (Fig. 2). Then the inverse value of the radius $r_{i}$ is an approximation of the curvature at $p_{i}$. Let $A$ denote the area of the triangle $\widehat{p_{i-1}} \widehat{p_{i} p_{i}}+1$ and $d_{i j}=\left\|p_{i}-p_{j}\right\|$. The discrete curvature $\kappa_{i}$ of the circle is given by:

$$
\kappa_{i}=1 / r_{i}=\frac{4 A}{d_{(i-1) i} d_{i(i+1)} d_{(i-1)(i+1)}}
$$

With the assumption of equidistance edges, and using the fact that the area of a triangle $\widehat{a b c}$ is $A=$ $\sqrt{s(s-a)(s-b)(s-c)}$ (where $s=\frac{a+b+c}{2}$ ) we get

$$
\kappa_{i}=\frac{2 \sqrt{d^{2}-d_{(i-1)(i+1)}^{2} / 4}}{d^{2}}
$$

Since $\gamma$ has a maximum curvature $\kappa$, therefore, $\kappa_{i} \leq \kappa$. In order that $\gamma_{p}$ satisfies the bounded curvature constraint we should have

$$
\left\|p_{i-1}-p_{i+1}\right\|=d_{(i-1)(i+1)} \geq L / n \sqrt{4-\frac{\kappa^{2} L^{2}}{n^{2}}}
$$

In summary, the $n+1$ ordered points $p_{0}, p_{1}, \ldots, p_{n} \in \Omega$, where $p_{0}=C, p_{n}=D$, form an equidistance polygonal curve with bounded curvature $\kappa$ and length $L$, if and only if, for all $i=1 \ldots n$, distances $\left\|p_{i-1}-p_{i}\right\|$ and $\left\|p_{i-1}-p_{i+1}\right\|$ satisfy (2) and (5). In the following section we try to find points $p_{0}, p_{1}, \ldots, p_{n} \in \Omega$, that satisfy constraints (2) and (5) by using multi-particle dynamical system approach.

\section{Path Planning Using Stable Multi-Particle SYSTEMS}

This section of the paper is organized as follows: In part A we consider path planning for single vehicle. In part B we address the multi-vehicle path planning problem. Finally we consider the problem of maximizing the coverage. Please note that in all of the cases we assume that there exist a dense set of solutions and we try to find one of them.

\section{A. Single Vehicle Path Planning}

1) Continuous Forces: Consider the waypoints of the vehicle as $p_{0}, \ldots, p_{n} \in \Omega$ as shown in (Fig. 1). Now lets assume that the points $p_{0}, \ldots, p_{n} \in \Omega$ are point mass moving particles with initial random distribution in the space. Also assume that the vector $F_{i}$ is a force acting on the $i-t h$ point. Therefore, we have

$$
m_{i} \ddot{p}_{i}=F_{i}
$$

Now assume $p_{0}=C, p_{n}=D$ are fixed, we can do this by assuming $m_{0}, m_{n} \rightarrow \infty$.

In the following we try to find vector forces $F_{i}, i=0 \ldots n$ in the way that the set of stable equilibria of the dynamical system (6) would be equal to the set of points $p_{0}, \ldots, p_{n}$ that satisfy constraints (2) and (5).

Define vector forces $F_{i}, i=0 \ldots n$ as follows:

$$
F_{i}=\sum_{\substack{j=0 \\ j \neq i}} f_{i j}\left(\left\|p_{i}-p_{j}\right\|\right) e_{i j}-g_{i}\left(\dot{p}_{i}\right)
$$

Where $e_{i j}=\frac{p_{j}-p_{i}}{\left\|p_{i}-p_{j}\right\|}$ is a unit vector from in the direction of $p_{i}$ to the $p_{j}$.

With the following conditions on functions $f_{i j}$ and $g_{i}$ :

1) $f_{i j}(x)=f_{j i}(x)$ for all $i$ and $j$

2) For any vector $P=\left(p_{0}, \ldots p_{n}\right) \in \Omega^{n+1}$, we have $f_{i j}\left(\left|p_{i}-p_{j}\right|\right)=0$ for all $i, j=0 \ldots n i \neq j$ if and only if, $p_{i}$ and $p_{j}$ satisfy (2) and (5) for all $i, j=0 \ldots n$.

3) Functions $f_{i j}$ are locally passive around their roots, i.e. if $f_{i j}\left(x_{0}\right)=0$ then

$$
\sigma f_{i j}\left(x_{0}+\sigma\right) \geq 0 \quad \forall \sigma \in\left[-\sigma_{0}, \sigma_{0}\right]
$$

4) $g(x)=0$ if and only if $x=0$

5) For all the vectors $x \in \mathbb{R}^{2}, x \cdot g_{i}(x) \geq 0$

6) Functions $f_{i j}$ and $g_{i}$ are continuous.

theorem 1: The vectors $P=\left(p_{0}, \ldots p_{n}\right) \in \Omega^{n+1}$ that satisfy (2) and (5) are asymptotical stable equilibria for the dynamical system (6). as follows:

Proof: For any $P=\left(p_{0}, \ldots p_{n+1}\right) \in \Omega^{n+1}$ we define $E(P)$

$$
E(P)=\sum_{j=i+1}^{n} \sum_{i=0}^{n-1} U_{i j}\left(\left\|p_{i}-p_{j}\right\|\right)+\frac{\|\dot{P}\|^{2}}{2}
$$

Where

$$
U_{i j}(\alpha)=\int_{\alpha_{0}}^{p} f_{i j}(\xi) d \xi
$$

and $\alpha_{0}$ is root of $f_{i j}$, i.e. $f_{i j}\left(\alpha_{0}\right)=0$.

Since function $f_{i j}$ is locally passive $U_{i j}(\alpha) \geq 0$; therefore

$$
E(p) \geq 0 \quad \forall P \in \Omega^{n+1}
$$

and

$$
\frac{\partial E}{\partial t}=\sum_{\substack{j=1 \\ j \neq i}}^{n} \sum_{i=1}^{n}<\frac{\partial U_{i j}\left(\left\|p_{i}-p_{j}\right\|\right)}{\partial p_{i}}, \dot{p}_{i}>+<\dot{P}, \ddot{P}>
$$




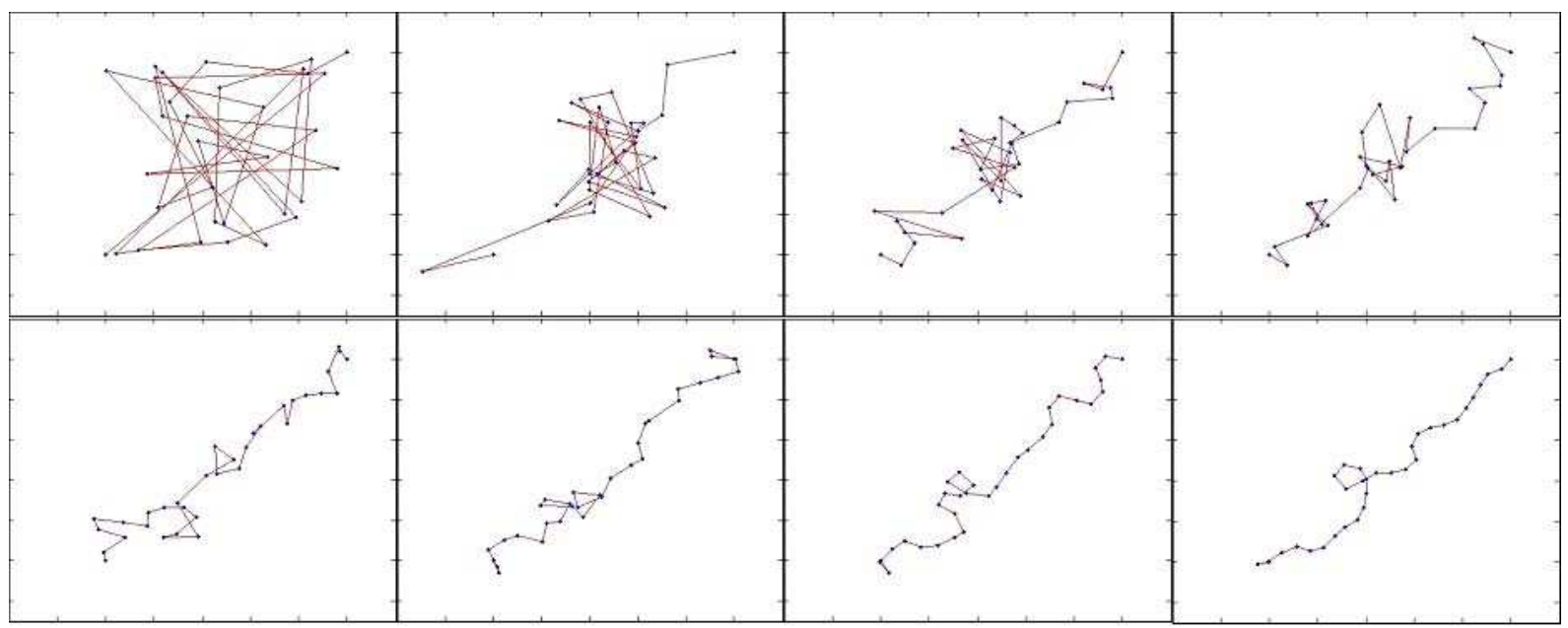

Fig. 3. An example of the convergence of multi particle system to a equilibrium which does not satisfy the curvature constraints (due to the sharp turn)

also

$$
\begin{gathered}
<\frac{\partial U_{i j}\left(\left\|p_{i}-p_{j}\right\|\right)}{\partial p_{i}}, \dot{p}_{i}>=<f_{i j}\left(\left\|p_{i}-p_{j}\right\|\right) \frac{\partial\left\|p_{i}-p_{j}\right\|}{\partial p_{i}}, \dot{p}_{i}> \\
=<f_{i j}\left(\left\|p_{i}-p_{j}\right\|\right) e_{j i}, \dot{p}_{i}>=<-f_{i j}\left(\left\|p_{i}-p_{j}\right\|\right) e_{i j}, \dot{p}_{i}>
\end{gathered}
$$

Using (6) and (7) in substituting $\ddot{P}$ we get

$$
\frac{\partial E}{\partial t}=-\sum_{i=1}^{n}<g\left(\dot{p}_{i}\right), \dot{p}_{i}>\leq 0
$$

The last inequality is from $x \cdot g_{i}(x) \leq 0$. So we get $\frac{\partial E}{\partial t} \leq$ 0 , hence from the Lyapunov theorem we can establish the stability of the dynamical system (6) also one can prove local asymptotic stability of the desired formation using LaSalle's invariance principle [9].

Please note that there are always additional unwanted equilibria for the dynamical system (6). Note that if one wishes to add restriction on the initial and final orientation, as well as the position of the vehicle, then this can be done by fixing $p_{1}$ and $p_{(n-1)}$ in addition to $p_{0}$ and $p_{n}$ (i.e. $m_{2}, m_{n-1} \rightarrow \infty$ ).

One example of the functions $f_{i j}$ and $g_{i}$ that satisfies the conditions of the theorem (1) is as follows:

$$
f_{(i-1) i}(\alpha)=k_{f}(\alpha-L / n)
$$

And

$$
f_{(i-1)(i+1)}(\alpha)=\left\{\begin{array}{c}
0 \text { if } \alpha \geq \eta \\
k_{f}(\alpha-\eta) \text { otherwise }
\end{array}\right.
$$

Where $\eta=L / n \sqrt{4-\frac{\kappa^{2} L^{2}}{n^{2}}}$ derived from the inequality (5). Also

$$
g_{i}(x)=v x
$$

It is easy to check that the above definitions of the functions $f_{i j}$ and $g_{i}$ satisfy the stability theorem conditions. But as we mentioned earlier there are additional equilibria for the dynamical system which are not desirable figure 3 shows the convergence of the multi-particle system to a equilibrium. But apparently the resulting curve does not satisfy the curvature constraint.

In the following we give an analysis of the net forces in the equilibrium which explains the failure of the method for in finding a desirable result. Consider a multi particle system consist of intermediate points $p_{1}, p_{2}, \ldots, p_{m}$ and fixed initial and final points $p_{0}, p_{f}$. Figure 4 shows the connectivity graph between the points, the points are connected iff there is a force between them. In any equilibrium the net force on the points is zero but as it has been dedicated in fig. 4 it is possible that the net force would be zero even though the forces are not zero (point $p_{3}$ ).

2) Discontinuous Forces: In the previous section we tried to convince the reader that by using continuous forces we will always have unfavorable equilibria. In this subsection we use discontinuous forces and by using net force analysis we show that an equilibrium is stable iff all of the forces are zero. Since we are using discontinues forces, we need nonsmooth analysis and stability of nonsmooth systems to analyse the dynamical system wit discontinuous right-hand sides but due to space limitation we omit this part and we refer the interested reader to the references [12]-[14].

Now consider the multi particle dynamical system defined in (6) and (7) with the same conditions on $f_{i j}$ and $g_{i}$ with exception that $f_{i j}$ could be discontinues in finite number of points, now we state the following theorem.

theorem 2 (Stability of discontinuous system): The vectors $P=\left(p_{0}, \ldots p_{n}\right) \in \Omega^{n+1}$ that satisfy (2) and (5) are asymptotical stable equilibria for the dynamical system (7). Now we define functions $g_{i}$ and $f_{i j}$ that satisfy the conditions of the theorem (2) as follows

$$
g_{i}(x)=v x
$$

and

$$
\begin{gathered}
f_{i(i+1)}(\alpha)=\left\{\begin{array}{cc}
w_{1} & \text { if }(\alpha-L / n) \geq w_{1} / k_{f} \\
k_{f}(\alpha-L / n) & \text { otherwise. } \\
-w_{1} & \text { if }(\alpha-L / n) \leq-w_{1} / k_{f}
\end{array}\right. \\
f_{(i-1)(i+1)}(\alpha)=\left\{\begin{array}{cc}
0 & \text { if } \alpha \geq \eta \\
w_{2} & \text { if } \alpha<\eta
\end{array}\right.
\end{gathered}
$$

Where $\eta=L / n \sqrt{4-\frac{\kappa^{2} L^{2}}{n^{2}}}$ is from the inequality (5). An unfavorable equilibrium is one that the net forces on the 


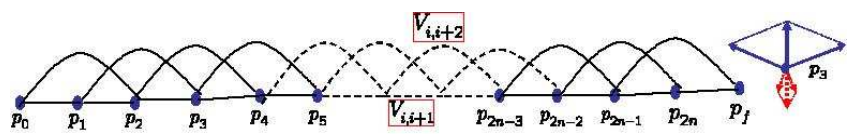

Fig. 4. The nodes represent the particles and the edges represent interaction forces between the particles.

particles are equal to zero but some of the forces are not equal to zero. Please note that all of the hard constrains (equidistance and curvature) are satisfied iff all of the forces are equal to zero. In the following theorem we show that with the forces defined in (19), (20) and with the key assumption that $w_{2}>2 w_{1}$ and even number of particles, there would be at most one unfavorable equilibrium which is unstable, therefore for almost all of the initial conditions the dynamical system converges to a favorable equilibrium.

theorem 3 (Instability of the unfavorable equilibrium): Consider a dynamical system of $2 n$ particles with the forces defined in (19) and (20) also assume that $L>\left\|p_{0}-p_{f}\right\|$ then there is at most one unfavorable equilibrium and and this equilibrium is unstable.

Proof: In the previous theorem we prove that the dynamical system is asymptotically stable so the state of the system converges to a stable equilibrium, in the equilibrium the net force on each particle is zero but not necessary all of the forces are zero. There are two type of forces, the forces that are between consecutive particles $V_{i, i+1}=f_{i, i+1}\left(\| p_{i}-\right.$ $\left.p_{i+1} \|\right) e_{i, i+1}$ for enforcing $\left\|p_{i}-p_{i+1}\right\|=L /(2 n+1)$ and curvature constraint derived forces $V_{i-1, i+1}=f_{i-1, i+1}\left(\| p_{i-1}-\right.$ $\left.p_{i+1} \|\right) e_{i-1, i+1}$.

We have

$$
\left\|V_{i, i+1}\right\| \leq w_{1}<w_{2} / 2
$$

also

$$
\left\|V_{i-1, i+1}\right\|=0 \text { or } w_{2}
$$

Now let construct a graph that nodes of the graph represent the particles and the edges represent the interaction forces (Fig. 4). Any particle except $p_{1}$ and $p_{2 n}$ is connected to 4 other particles (note that $p_{0}$ and $p_{f}$ are not moving particles). At node $p_{1}$ there are 3 forces $V_{0,1}, V_{1,2}$ and $V_{1,3}$, since the net force is zero therefore

$$
V_{0,1}+V_{1,2}+V_{1,3}=0
$$

So

$$
\left\|V_{0,1}+V_{1,2}\right\|=\left\|V_{1,3}\right\|
$$

Now we claim that $V_{1,3}=0$ otherwise we have $V_{1,3}=w_{2}$ so

$$
\left\|V_{0,1}+V_{1,2}\right\|=\left\|V_{1,3}\right\|=w_{2}
$$

But we have

$$
\left\|V_{0,1}+V_{1,2}\right\| \leq\left\|V_{0,1}\right\|+\left\|V_{1,2}\right\| \leq 2 w_{1}<w_{2}
$$

Which is a contradiction, therefore $V_{1,3}=0$. Since the edge $V_{1,3}$ is eliminated now there are 3 edges connected to node $p_{3}$ which are $V_{2,3}, V_{3,4}$ and $V_{3,5}$, with the similar argument one can show that $V_{3,5}=0$, similarly $V_{5,7}=0, \ldots, V_{2 i-1,2 i+1}=$ $0, \ldots, V_{2 n-1, f}=0$. So all of the curvature constraint driven forces connecting odd edges are eliminated. Now one can use the exact same argument this time starting from node $p_{2 n}$, since $p_{2 n}$ connected to 3 nodes, one could conclude $V_{2 n, 2 n-2}=0$ and similarly $V_{2 n-2,2 n-4}=0$ and $V_{2 n-4,2 n-6}=$ $0, \cdots$. Now all of the curvature constraint driven forces are eliminated and the only way that $V_{i, i+1} \neq 0$ with the net force equal to zero is that all of the particles are in a line connecting $p_{0}$ and $p_{f}$ which is clearly unstable.

Please note that if $L \leq\left\|p_{0}-p_{f}\right\|$ there would be just one equilibrium (which is stable) and which is the configuration that all of the particles are in a line connecting $p_{0}$ and $p_{f}$.

Figure (5) shows an example of a fixed length bounded curvature trajectory connecting two points generated by 100 particles and the initial conditions are chosen randomly.

\section{B. Multi-Vehicles}

In the case of multiple vehicles, the structure of the dynamical system is exactly the same as in single vehicle case, with the exemption that, we need to add extra terms to the forces in order to avoid the collision between vehicles.

Consider two vehicles with speeds $v_{1}$ and $v_{2}$ that travel distances $L_{1}$ and $L_{2}$. The corresponding waypoints for the two vehicles are $p_{0}, p_{1}, \ldots, p_{n}$ and $q_{0}, q_{1}, \ldots, q_{m}$. Collision between two vehicles can occurs if two vehicles arrive to the same point simultaneously. Collision happens if for some $i, j$ the following two conditions are satisfied :

- Simultaneous timing condition:

$$
\left\|i \frac{L_{1}}{n v_{1}}-j \frac{L_{2}}{m v_{2}}\right\| \leq \varepsilon_{1}
$$

- Passing through the same point condition:

$$
\left\|p_{i}-q_{j}\right\| \leq \varepsilon_{2}
$$

Thus in order to enforce collision free trajectories, we add a new interaction force between two waypoints $p_{i}$ and $q_{j}$ whenever $i, j$ satisfy (27). Therefore:

$$
f_{i j}(\alpha)=\left\{\begin{array}{c}
0 \text { if } \alpha \geq \varepsilon_{2} \\
w_{3} \text { otherwise }
\end{array}\right.
$$

please note that the interaction force defined in (29) unlike the previous forces is between two different trajectories. It is also clear that (29) satisfies the stability theorems conditions.

Likewise the single vehicle case, by adding forces (29), dynamical system (6) will have extra unwanted equilibria. Fortunately these equilibria are unstable based on the following theorem.

theorem 4 (Instability of unfavorable equilibria):

Assume that there are $V$ vehicles and the trajectory of each vehicle is generated by $2 n_{i} i=1 \ldots V$ particles and the forces are defined as Eq. (19), (20) and (29) with the following constraints on $w_{1}, w_{2}$ and $w_{3}$

$$
\begin{gathered}
2 w_{1}<w_{2} \\
2\left(w_{1}+w_{2}\right)<w_{3}
\end{gathered}
$$

and with the assumption that not more than two vehicles could collide at the same location and time, then all of the unwanted equilibria are unstable. 

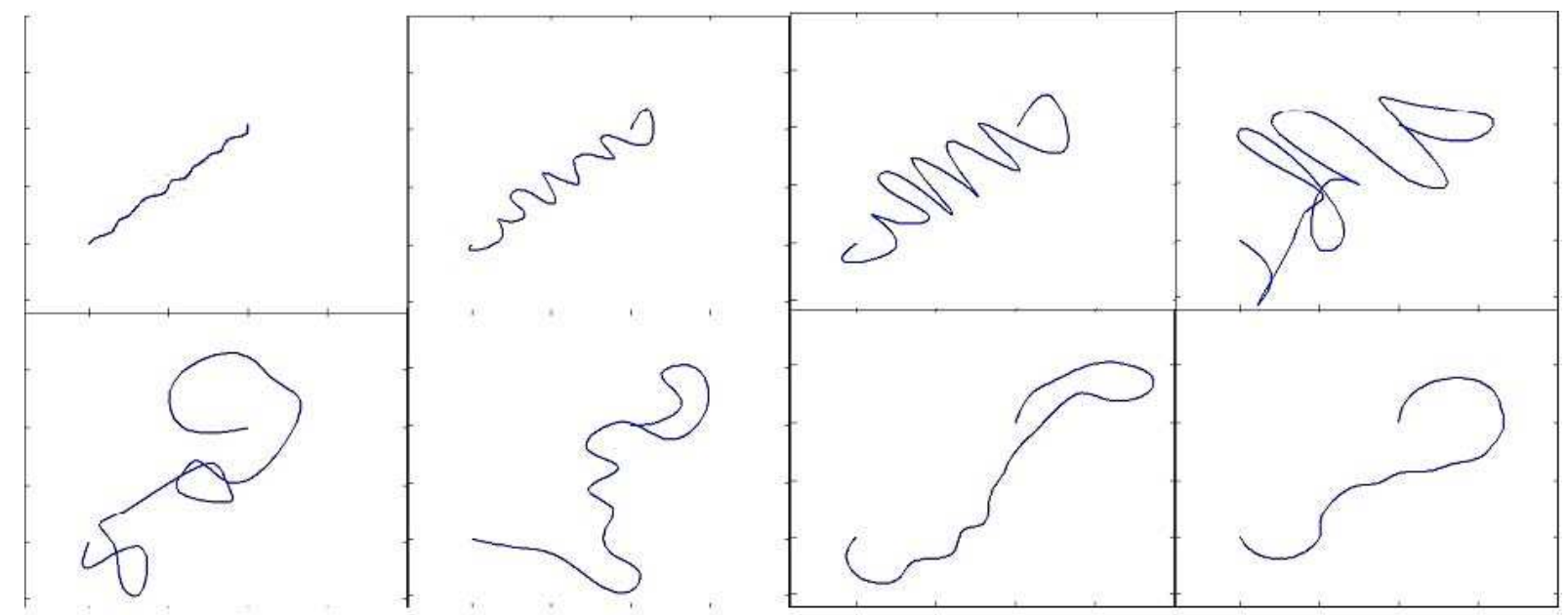

Fig. 5. An example of a fixed length bounded curvature trajectory connecting two points generated by 100 particles.

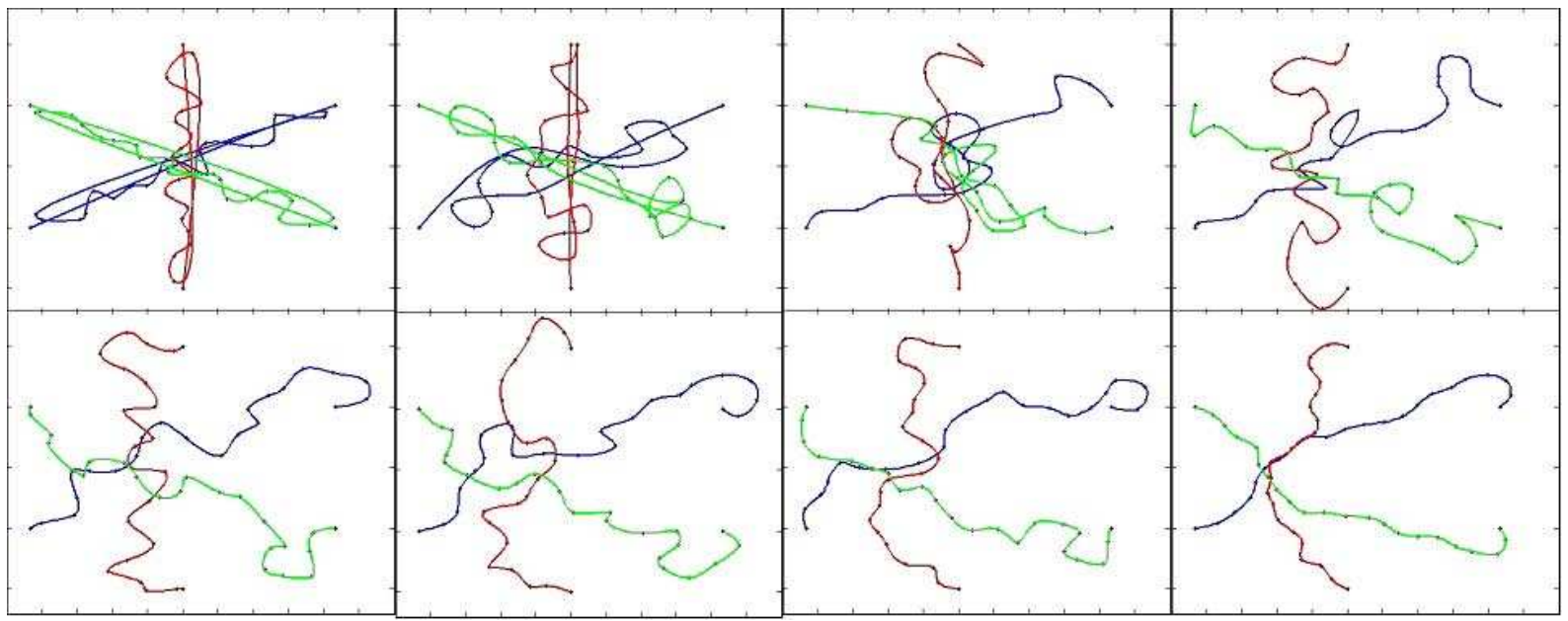

Fig. 6. Example of Multi-vehicle path planning with collision avoidance.

Proof: Fist we prove that in any equilibrium the trajectories will be collision free pathes then one could use the results from single vehicle case to prove that stable equilibria are all favorable. Assuming the two vehicles collide at a point represented by $p_{i}$ and $p_{k}$, there will be at most 6 forces on the particle $p_{i}$.

- Collision avoidance force as $V_{i, k}=w_{3} e_{i, k}$

- Equidistance driven forces as $V_{i-1, i}=f_{i-1, i} e_{i-1, i}$ and $V_{i, i+1}=f_{i, i+1} e_{i, i+1}$

- Curvature forces as $V_{i, i+2}=f_{i, i+2} e_{i, i+2}$ and $V_{i-2, i}=$ $f_{i-2, i} e_{2-1, i}$

Since net force is zero therefore

$$
V_{i-1, i}+V_{i, i+1}+V_{i-2, i}+V_{i, i+2}+V_{i, k}=0
$$

and

$$
\left\|V_{i-1, i}+V_{i, i+1}+V_{i-2, i}+V_{i, i+2}\right\|=\left\|V_{i, k}\right\|=w_{3}
$$

But

$$
\begin{gathered}
\left\|V_{i-1, i}+V_{i, i+1}+V_{i-2, i}+V_{i, i+2}\right\| \leq \\
\left\|V_{i-1, i}\right\|+\left\|V_{i, i+1}\right\|+\left\|V_{i-2, i}\right\|+\left\|V_{i, i+2}\right\| \\
\leq w_{1}+w_{1}+w_{2}+w_{2}=2\left(w_{1}+w_{2}\right)<w_{3}
\end{gathered}
$$

Which is contradiction with equality in eq. (32) and $V_{i, k}=0$

Therefore in the equilibrium all trajectories are collision free and there are no forces between two separate trajectories. But in theorem (6) we proved that in this case all of the stable equilibria are favorable.

Figure 6 depicts an example of multi vehicle path planning with collision avoidance using 3 vehicles.

\section{Coverage Maximization}

Assume that each vehicle equipped with a sensor which has a fixed sensing radios. We would like to generate trajectories that maximize spatio-temporal coverage while satisfying hard constraints such as collision avoidance and specifications on initial and final positions Since the length of the trajectories are fixed, one way of maximizing the overall coverage is to minimize the intersection of field of view of waypoints.

Assume that two way points $p_{i}$ and $q_{j}$ have field of views with radii $r_{i}$ and $r_{j}$, in order to maximize the total coverage we can minimize the intersection by trying to enforce $\| p_{i}-$ $q_{j} \| \geq r_{i}+r_{j}$. we can add following interaction force to the 


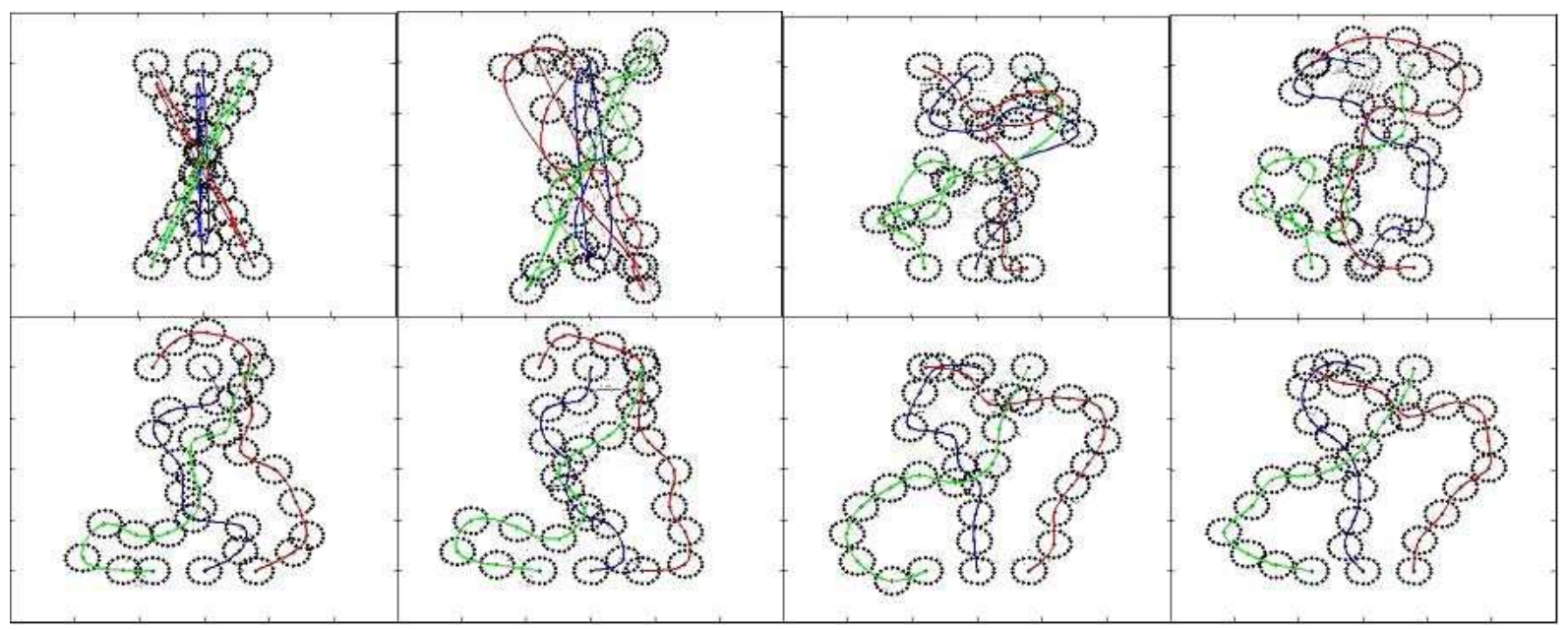

Fig. 7. Simulation of 3 vehicles path planing while locally maximizing the coverage

dynamical system (6).

$$
f_{i j}(\alpha)=\left\{\begin{array}{c}
0 \quad \text { if } \alpha \geq r_{i}+r_{j} \\
w_{0} \frac{\alpha-r_{i}-r_{j}}{\left\|r_{i}+r_{j}\right\|} \text { otherwise }
\end{array}\right.
$$

Adding these new interaction forces to the system may introduce new undesirable stable formation as well. One can prove that this can be prevented by setting stiffness $w_{0}$ much less than $w_{1}$.

Figure (7) shows an example of coverage maximization by 3 vehicle. Please note that the generated trajectories are local optimal solutions.

\section{Conclusion}

In this paper we presented a path planning algorithm for time sensitive cooperative surveillance using autonomous vehicles equipped with sensors. The main challenges were to generate feasible trajectories while satisfying hard constraint such as collision avoidance and specifications on initial and final positions. Our approach is based on approximation of the trajectories of vehicles using sequence of waypoints and treating each way point as a moving particle in the space. We defines interaction forces between the particles such that the resulting multi-particle system will be stable, moreover, the trajectories generated by the waypoints in the equilibria of the multi-particle system will satisfy all of the hard constraint while generating a suboptimal solution to the coverage problem.

\section{REFERENCES}

[1] L. E. Dubins, On curves of minimal length with a constraint on average curvature, and with prescribed initial and terminal positions and tangents, Amer. J. Math., vol. 79, no. 3, pp. 497-516, 1957

[2] D. A. Castanon, Approximated dynamic programming for sensor management, in Proc. IEEE Conf. Decision Control, San Diego, CA, pp. 1202-1207. Dec. 1997

[3] D. J. Cool, P. Gmytrasiewicz, and L. B. Holder, Decision-theoretic cooperative sensor planning, IEEE Trans. Pattern Anal. Machine Intell., vol. 18, no. 10, pp. 892-902, Oct. 1996.

[4] M. Kalandros, L. Y. Pao, and Y. Ho, "Randomization and superheuristics in choosing sensor sets for target tracking applications, in Proc. IEEE Conf. Decision Control, vol. 2, Phoenix, AZ, pp. 18031808. Dec. 1999
[5] J. Cortes, S. Martinez, T. Karatas, and F. Bullo, Coverage control for mobile sensing networks. In Proceedings of IEEE International Conference on Robotics and Automation, 2002, pp. 1327-1332.

[6] J.J. Enright and E. Frazzoli and K. Savla and F. Bullo. On Multiple UAV Routing with Stochastic Targets: Performance Bounds and Algorithms. Proc. of the AIAA Conf. on Guidance, Navigation, and Control, August 2005.

[7] M.P. Do Carmo, Differential geometry of curvs and surfaces, Prentice Hall, 1976.

[8] V.I. Arnold, Mathematical Methods of Classical Mechanics, Springer, 2000.

[9] S. Sastry, Nonlinear Systems, Springer, 2004.

[10] A. Ahmadzadeh, J. Keller, G. Pappas, A. Jadbabaie and V. Kumar, Multi-UAV Deployment for Coverage with Spatio-Temporal Specifications IEEE Conference on Decision and Control 2006

[11] A. Ahmadzadeh, J. Keller, G. Pappas, A. Jadbabaie and V. Kumar, An Optimization-based Approach to Time Critical Cooperative Surveillance and Coverage with Unmanned Aerial Vehicles, In International Symposium on Experimental Robotics 2006

[12] F.H. Clarke, Yu.S. Ledyaev, R.J. Stern, and P.R. Wolenski. Nonsmooth Analysis and Control Theory. Graduate Texts in Mathematics; 178. Springer, New York, 1998.

[13] A. F. Filippov. Differential equations with discontinuous right-hand side. Mathematics and Its Applications (Soviet Series). Kluwer Academic Publishers, The Netherlands, 1988.

[14] D. Shevitz and B. Paden. Lyapunov stability theory of nonsmooth systems. IEEE Transactions on Automatic Control, 39(9):19101914,1994. 